based upon how important they felt it was in determining treatment success culminating in a final consensus meeting. Stakeholders were recruited nationally and grouped into three panels (Surgeons and Radiologists, Gastroenterologists and IBD specialist nurses, Patients). Participants received feedback from their panel (in the second round) and all participants (in the third round) to allow refinement of their scores.

Results A total of 295 outcomes were identified from systematic reviews and interviews that were categorised into 92 domains. 187 stakeholders (response rate 78.5\%) prioritised 49 outcomes through a three-round Delphi study.

The final consensus meeting of 41 experts and patients generated agreement on an eight domain COS. The COS comprised three patient-reported outcome domains (quality of life, incontinence and a combined score of patient priorities) and five clinician-reported outcome domains (perianal disease activity, development of new perianal abscess/sepsis, new/recurrent fistula, unplanned surgery and faecal diversion).

Conclusion A fistulising pCD COS has been produced by all key stakeholders. Application of the COS will reduce heterogeneity in outcome reporting, thereby facilitating more meaningful comparisons between treatments, data synthesis and ultimately benefit patient care.

\section{OWE-010 BACTERIAL AND FUNGAL COMMUNITIES IN FAECES AND BIOPSIES IN IBD}

${ }^{1}$ Alessandra Frau*, ${ }^{2}$ Umer Zeeshan ljaz,. ${ }^{1}$ Barry Campbell,. ${ }^{1}$ Alistair Darby, ${ }^{1}$ John Kenny, ${ }^{3}$ Neil Hall, 'Chris Probert. 'University Of Liverpool, Liverpool, UK; ${ }^{2}$ University of Glasgow, Glasgow, UK; ${ }^{3}$ Earlham Institute, Norwich, UK

\subsection{6/gutjnl-2018-BSGAbstracts.115}

Introduction Crohn's disease (CD) is associated with a dysbiosis of the bacterial microbiome: few studies have looked at fungi (the mycobiome). Disturbance of Candida and Saccharomyces communities have been found and anti-saccharomyces cerevisiae antibodies (ASCA) may occur in CD. We found fungal metabolites in faeces of CD patients. We aimed to characterise the fungal and bacterial communities in the lumen and mucosa in IBD.

Methods Faeces was obtained from 63 donors (23 CD, 20 UC, 20 controls). Biopsies from the ileum and colon were obtained from 40 donors (18 CD, 12 UC, 10 controls). Metagenomic DNA was extracted and used for fungal 18S rRNA and bacterial 16S rRNA PCR. Amplicons were sequenced using Illumina MiSeq. Reads were quality filtered, trimmed, paired and OTUs were clustered. OTU table, fasta file, phylogenetic tree and metadata were used for statistical analysis. ASCA and calprotectin were measured by ELISA.

Result The faecal bacterial community is dysbiotic in CD; alpha diversity is low (figure 1), with beta diversity CD samples form a separate cluster (figure 2) and these are more phylogenetically conserved (figure 3). Disease distribution, ASCA and calprotectin explain some of the diversity. The mycobiome does not seem to be different in the faeces in CD and most samples have a high abundance of Saccharomyces. Other yeasts and moulds were also seen. Inter-kingdom cooccurrence network analysis shows few main clusters dominated by Firmicutes, but also Bacteroidetes and Actinobacteria, and two fungi: Malassezia and Aspergillus.

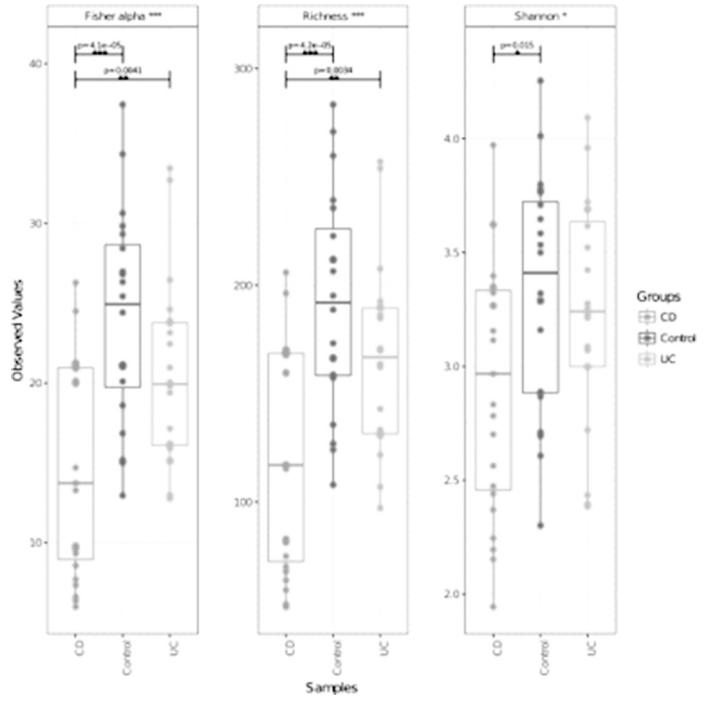

Abstract OWE-010 Figure 1 Alpha diversity of bacteria in stool

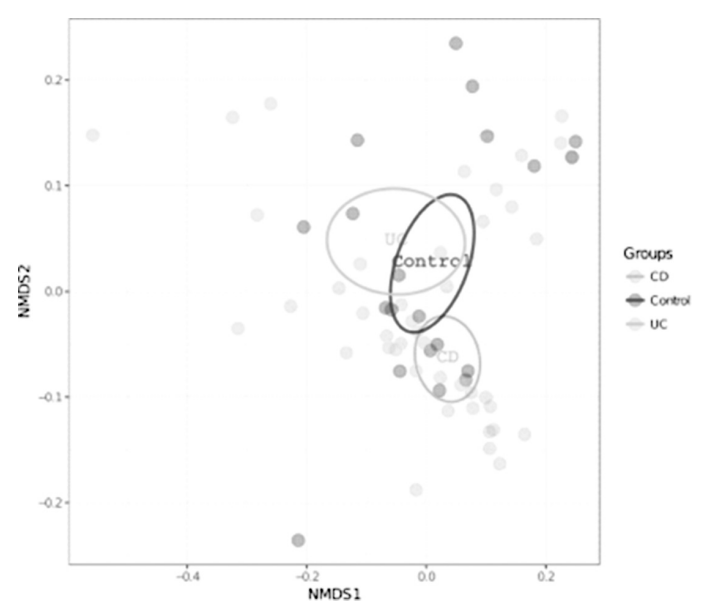

Abstract OWE-010 Figure 2 Nonmetric distance scaling (NMDS) weighted UniFrac (bacteria-stool) $R^{2}=0.09 n=-0.004$

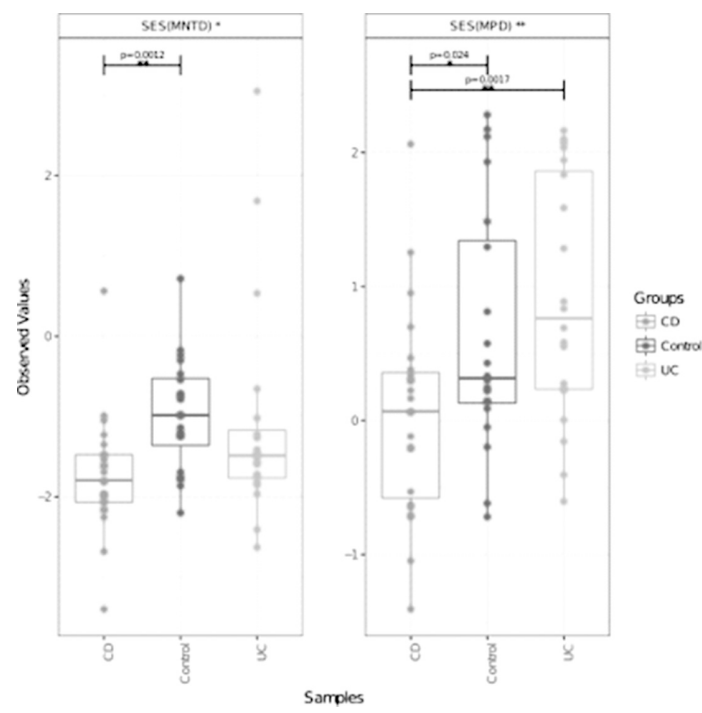

Abstract OWE-010 Figure 3 Phylogenetic dispersion (bacteria-stool) 


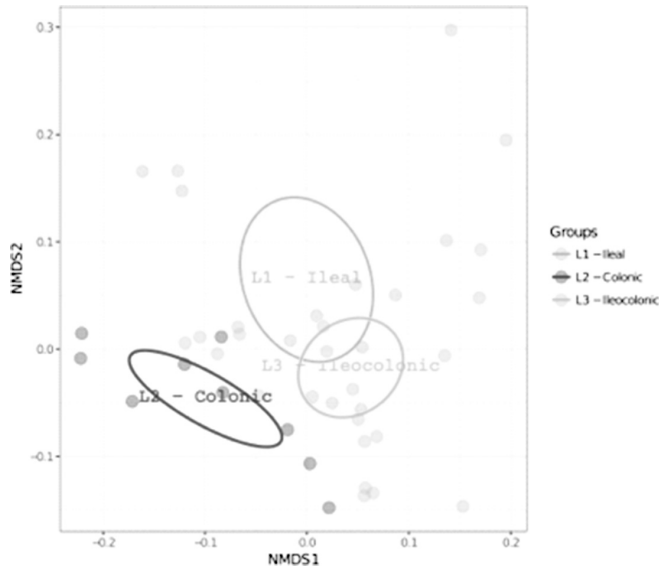

Abstract OWE-010 Figure 4 NMDS weighted UniFrac (bacteriabiopsies - disease distribution) $R^{2}=0.17 p=0.001$

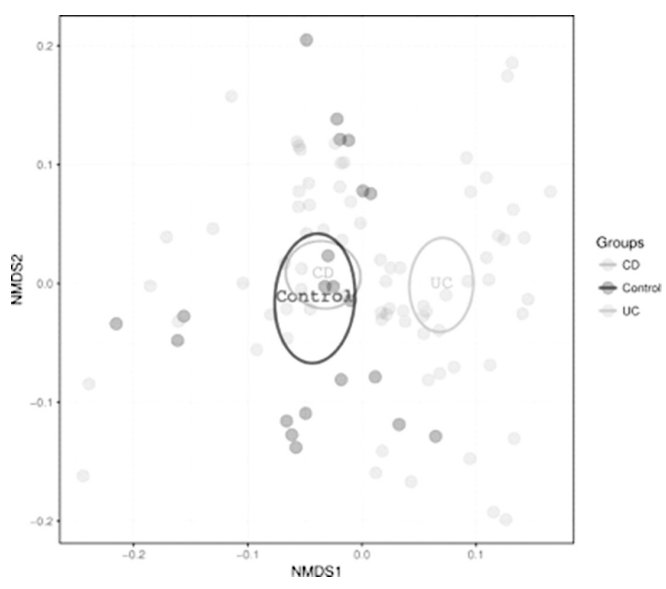

Abstract OWE-010 Figure 5 NMDS weighted UniFrac (bacteria-all biopsies - diagnosis) $R^{2}=0.11 p=0.001$

The mucosal bacterial community was dysbiotic and influenced by subject, disease distribution (figure 4) and diagnosis (figure 5). In terms of mycobiome, fewer viable reads were obtained, due to limited template. Saccharomyces was the most abundant fungi, but it was absent in some samples, other relevant genera were Malassezia, Cladosporium, Aspergillus and Candida. The last was found more often in controls.

Conclusions CD patients' bacterial community is dysbiotic but fungi are not. Saccharomyces dominated most of faecal samples, but not biopsies. This implies it may not be a permanent resident of the mucosae. Fungi may arise from food: it is hard to discriminate what comes from food and what is active in the gut. The concept of a resident, symbiotic mycobiome needs further exploration.

\section{OWE-011 CLINICAL EFFECTIVENESS, SAFETY AND IMMUNOGENICITY OF ANTI-TNF THERAPY IN CROHN'S DISEASE: 12-MONTH DATA FROM PANTS}

${ }^{1}$ Nicholas Kennedy*, ${ }^{1,2}$ Graham Heap, ${ }^{1}$ Gareth Walker, ${ }^{1}$ Claire Bewshea, ${ }^{2}$ Sonia Bouri, ${ }^{1}$ James Goodhand, PANTS investigator consortium, 'Tariq Ahmad. 'IBD Pharmacogenetics, University of Exeter, Exeter, UK; ${ }^{2}$ Abbvie, Chicago, USA; ${ }^{3}$ BBD Unit, St Mark's Hospital, London, UK

10.1136/gutjil-2018-BSGAbstracts.116
Introduction PANTS (Personalised Anti-TNF Therapy in Crohn's disease [CD]) is a 3 year prospective observational UK-wide study investigating primary non-response (PNR), loss of response and adverse drug reactions to infliximab (IFX: Remicade [REM], CT-P13) and adalimumab (ADL: Humira). We now report the wk 54 clinical effectiveness and safety outcomes, and immunogenicity data to date.

Methods Inclusion criteria included: CD patients aged $\geq 6$ years, active inflammatory disease (raised CRP [>3 mg/L] or calprotectin $[\geq 50 \mu \mathrm{g} / \mathrm{g}])$ and no prior anti-TNF therapy. PNR was defined at wk $12-14$ as a requirement for ongoing steroids, or both HBI failed to fall by $\geq 3$ points or to $\leq 4$ and CRP failed to fall by $\geq 50 \%$ or to $\leq 3 \mathrm{mg} / \mathrm{L}$. Remission was defined at wks 14 and 54 as $\mathrm{HBI} \leq 3$ points and CRP $\leq 3 \mathrm{mg} / \mathrm{L}$ and no concomitant steroids. Patients who stopped drug other than for elective withdrawal, pregnancy or loss to follow-up were regarded as treatment failures for subsequent endpoints. Drug (DL) and anti-drug antibody (ADA) levels were measured using the IDKmonitor drug tolerant assays. Immunogenicity was defined as ADA titre $\geq 10 \mathrm{AU} / \mathrm{ml}$ + undetectable DL.

Results 1601 (49\% male, median age 33 years [IQR 23-47]) eligible patients were recruited from 118 sites. Patients were treated with IFX (751 [47\%]: REM, 200 [12\%] CT-P13) or ADL (650 [41\%]). Baseline characteristics included: median disease duration 3 years (IQR 1-10); steroids 27\%, azathioprine 44\%, mercaptopurine $8 \%$, methotrexate 5\%; median $\mathrm{CRP}$ in IFX $9 \mathrm{mg} / \mathrm{L}$ (IQR CI 3-24) and $6 \mathrm{mg} / \mathrm{L}$ (IQR 2-14) in ADL. PNR at week $12-14$ was $21 \%, 21 \%$ and $26 \%$ in the REM, CT-P13 and ADL treated patients respectively. PNR was associated with older age $(p=0.0004)$, higher BMI $(p=0.03)$ and low DL $(\mathrm{p}<0.0001$ for IFX and ADL). Week 54 remission rate was $40 \%, 40 \%$ and $34 \%$ of the REM, CT-P13 and ADL treated patients. At wk 54, the immunogenicity rate for REM, CT-P13 and ADL was 26\%, 28\% and $11 \%$ rising to $42 \%, 38 \%$ and $23 \%$ by 3 years respectively (IFX vs. ADL $\mathrm{p}<0.0001$, REM vs. CT-P13 $\mathrm{p}=0.25)$. Immunogenicity was associated with non-remission at wk $54(\mathrm{p}<0.0001$ for both IFX and $\mathrm{ADL})$. Immunomodulator use reduced the risk of immunogenicity for both IFX $(\mathrm{HR}=0.37, \mathrm{p}<0.0001)$ and ADL $(\mathrm{HR}=0.34, \mathrm{p}<0.0001) .140$ patients $(9 \%)$ withdrew drug for SAEs including 5 who died, 3 from CD and 2 from possibly drug-related acute respiratory illness.

Conclusions This is the largest prospective real-life study of anti-TNF therapy in IBD. We report the clinical effectiveness, safety and immunogenicity of REM, CT-P13, and ADL. This cohort provides a unique bioresource for multi-omic studies investigating personalised approaches to anti-TNF therapy.

\section{OTH-003 PAEDIATRIC CROHN'S DISEASE PATIENTS IN REMISSION HAVE A REDUCED SKELETAL MUSCLE PROTEIN BALANCE AFTER FEEDING}

${ }^{1,2}$ Gordon Moran, ${ }^{3}$ Amanda Walker, ${ }^{3}$ Aline Nixon, ${ }^{1}$ David Devadason, ${ }^{4}$ Rafeeq Muhammed,
${ }^{3}$ Kostas Tsintzas, ${ }^{1}$ Sian Kirkham, ${ }^{5}$ Francis Stephens. ${ }^{1}$ Nottingham University Hospitals NHS
Trust; ${ }^{2}$ NIHR Nottingham Biomedical Research Centre; ${ }^{3}$ School of Life Sciences, University of
Nottingham; ${ }^{4}$ Birmingham Children's Hospitals; ${ }^{5}$ Sports and Health Sciences, University of
Exeter

10.1136/gutjnl-2018-BSGAbstracts. 117

Introduction Sarcopenia is common in active Crohn's disease (CD) and still prevalent in remission. This can lead to fatigue, physical inactivity and poor quality of life but the aetiology is 